ter. Is disclosure of potential conflicts of interest in medicine and public health sufficient to increase transparency and decrease corruption? J Epidemiol Community Health. 2009;63(8):603-5.

5. World Health Organization. Standards and operational guidance for ethics review of health-related research with human participants. Geneva: WHO; 2011.

6. Sierra R. Quién protege a los conejos [Internet]. San José, Costa Rica: La Nación; 2011 [citado el 22 de enero de 2012]. Disponible en: http://www.nacion.com/2011-11-24/ Opinion/quien-protege-a-los-conejos.aspx

Correspondencia: Rafaela Sierra

Dirección: Apartado 1374-1250. San José, Costa Rica.

Teléfono: (506) 25831962

Correo electrónico: rafaela.sierra@ucr.ac.cr

\section{COMENTARIOS AL ARTÍCULO "ÉTICA E INVESTIGACIÓN"}

\section{COMMENTS TO THE ARTICLE "ETHICS AND RESEARCH}

\author{
Sergio Eduardo Gonorazky ${ }^{1, a}$
}

Sr. Editor. En el artículo de Zavala y Alfaro-Mantilla ${ }^{(1)}$ se vierten algunas expresiones que llaman al debate, tal como los autores invitan a hacerlo.

Cuando manifiestan que los ensayos clínicos multicéntricos actualmente: se llevan a cabo exitosamente, sin objeciones éticas y con ventajas para pacientes, instituciones e investigadores, dan por ciertas varias hipótesis que deben demostrarse.

La expresión "exitosamente" es equívoca. Puede significar que se ha demostrado la eficacia de la droga experimental o qué, más allá del resultado, se pudo completar adecuadamente el protocolo, en tiempo y forma. Son numerosos los estudios en fase III que finalizaron y finalizan sin demostración de eficacia y otros que debieron suspenderse ante la evidencia de reacciones adversas a la droga que superaban los beneficios pretendidos, lo que por otra parte no debe sorprendernos de un ensayo clínico. Sin embargo, si se pretende en una generalización que no admite excepciones, afirmar que el desarrollo de los protocolos

\footnotetext{
1 Departamento de Investigación, Hospital Privado de Comunidad. Mar del Plata, Argentina.

a Médico neurólogo
}

Recibido: 22-02-12 Aprobado: 07-03-12 es exitoso pues estos se efectuaron y efectúan técnica y éticamente de acuerdo con lo pautado, la afirmación es falsa. En Argentina, y este es solo un ejemplo entre otros, acaba de ser sancionado el laboratorio Glaxo y varios investigadores por distintas infracciones procedimentales y éticas en el transcurso del estudio COMPAS (el ensayo tenía por objetivo evaluar la eficacia de una vacuna destinada a la prevención de la neumonía y otitis media debida a infección por neumococo) ${ }^{(2)}$.

La supuesta ausencia de objeciones éticas revela más la falta de transparencia en la información y en los mecanismos de control que en la optimista afirmación de los autores. En este momento se está llevando adelante en Perú un estudio, el BAY12-8039 que fue objetado por razones metodológicas y éticas por el Comité de Ética de nuestra institución (Hospital Privado de Comunidad, Mar del Plata, Argentina). Este rechazo no se anuncia como tal en la página de www.clinical. trials.gov sino que, por el contrario, allí figura como que el estudio comenzó y finalizó prematuramente en nuestro nosocomio lo que se contradice con lo arriba expresado ${ }^{(3)}$. Más allá de la pertinencia o no del rechazo, el hecho demuestra tres cosas: que se están llevando adelante estudios que tienen objeciones éticas, que no hay transparencia y que tampoco hay veracidad en la información, aun en organismos de alto prestigio internacional.

Quien suscribe publicó en el año 2008 un trabajo en el que evaluó y discutió la eficiencia y supuesta independencia de los comités de ética de investigación no institucionales que desempeñan su actividad con fines lucrativos y que aprueban protocolos patrocinados por la industria (y son contratados y pagados por ella para efectuarlo) ${ }^{(4)}$. En dicho informe se determinó una mediana de tres objeciones relevantes en treintitres protocolos analizados y aprobados por estos comités para ser realizados en Argentina. Una editorial de la revista Nature ${ }^{(5)}$ denuncia en igual sentido los "errores" que comenten los comités de ética en investigación que evalúan protocolos con fines de lucro.

También es equívoca la expresión que la investigación es beneficiosa para los pacientes. En Argentina es frecuente escuchar que en un estudio de investigación los primeros beneficiarios son los pacientes. En un escrito de la Cámara Argentina de Especialidades Medicinales (que representa a la industria farmacéutica extranjera) se expresa que: Los primeros beneficiarios de este proceso [de investigación] son los pacientes ya que acceden a tratamientos de última generación que incluso, aún no están disponibles [y que] quienes participan de un estudio clínico tienen acceso a 
prestaciones de alto estándar de calidad a nivel de intervenciones $y$ atención profesional durante y posterior al desarrollo del estudio. Además existe acceso temprano y prolongado a medicamentos que pueden cambiar por completo el pronóstico de su enfermedad ${ }^{(6)}$. La falsedad de esta expresión es manifiesta pues justamente lo que se pretende probar es si el tratamiento es eficaz o no. Otros expresan que los pacientes están mejor atendidos durante la investigación. Si los pacientes no estaban recibiendo una atención adecuada previo a la investigación, debe considerarse que esta se está realizando sobre población vulnerable y que la obligación del investigador y el patrocinador es seguir brindándole la mejor atención probada en el estudio una vez finalizado (ver Declaración de Helsinki 2008), lo que no es lo que suele ocurrir.

Es claro, como manifiestan los autores, que la ganancia es la que determina la conducta del inversionista. Quizá llegó el momento de discutir si la investigación biomédica debe estar determinada y legitimada por la lógica del lucro o debemos evaluar la posibilidad de discutir un sistema cuya conducta se determine por las necesidades de los pueblos.

\section{Conflictos de interés}

El autor declara no tener ningún conflicto de interés.

\section{REFERENCIAS BIBLIOGRÁFICAS}

1. Zavala S, Alfaro-Mantilla J. Ética e investigación. Rev Peru Med Exp Salud Publica. 2011;28(4):664-9.

2. Administración Nacional de Medicamentos, Alimentos y Tecnología Médica. ANMAT amplía información sobre el fallo del juez Aguinsky que ratifica lo actuado por el estado nacional [Internet]. Buenos Aires; ANMAT; 2012 [citado el 5 febrero de 2012]. Disponible en: http://www.anmat.gov.ar/ comunicados/Comunicado COMPASS.pdf

3. ClinicalTrials.gov. Moxifloxacin in Pediatric Subjects With Complicated Intra-abdominal Infection (MOXIPEDIA). [Internet] Bethesda: A service of the U.S. National Institutes of Health; 2012 [citado el 5 de febrero de 2012] Disponible en: http://www.clinicaltrials.gov/ct2/show/study/NCT01 069900?term=moxifloxacin+AND+bayer\&rank=1\&show_ locs $=$ Y\#locn

4. Gonorazky SE. Comités de ética independientes para la investigación clínica en La Argentina. Evaluación y sistema para garantizar su independencia. Medicina (Buenos Aires). 2008;68(2):113-9.

5. Who watches the watchmen? Nature 2011;476(7359):125.

6. Estudios Clínicos. Industria sin chimeneas. CAEME. 2010;(1):32-9.

Correspondencia: Sergio Eduardo Gonorazky

Dirección: Córdoba 4545. Mar del Plata, Argentina.

Teléfono: (54) 2234990000

Correo electrónico: egonora@gmail.com

\section{COMENTARIOS AL ARTÍCULO "ÉTICA E INVESTIGACIÓN" - RÉPLICA DE AUTORES}

\section{COMMENTS TO THE ARTICLE "ETHICS AND RESEARCH" - AUTHORS' REPLY}

\section{Salomón Zavala1,2,a Julio Alfaro-Mantilla ${ }^{1,3, b}$}

Cuando decimos en nuestro artículo que los estudios de investigación se realizan exitosamente, queremos expresar que se cumplen los objetivos generales, no que son perfectos o que no tienen fallas, tal como diríamos por ejemplo que el Mundial de Futbol Sudáfrica 2010 se desarrolló exitosamente o que nuestras pasadas elecciones generales fueron exitosas porque ya tenemos nuevo presidente, nadie las ha impugnado y el ganador ha sido proclamado por el Jurado Nacional de Elecciones. Nos disculpamos por no haber sido más explícitos.

Nuestro artículo, que es de opinión, no es exhaustivo y recoge o describe la experiencia nacional. Hemos tenido mucho cuidado de no pronunciarnos sobre lo que no nos consta.

En ese sentido, cuando se dice que las regulaciones en los países en desarrollo pueden ser más laxas no nos damos por aludidos porque en nuestro país existe el Instituto Nacional de Salud (INS), cuyo "Reglamento de Ensayos Clínicos" (1) está ajustado a las Normas Internacionales y estas se reflejan a su vez en nuestros Reglamentos y Manuales de Normas Procedimientos. Nuestro problema, en todo caso, es que tenemos que funcionar con el celo del primer mundo pero con las facilidades, y a veces los obstáculos, del tercer mundo.

Los ensayos clínicos en nuestro medio se llevan a cabo mayormente en los hospitales de Ministerio de Salud o de la Seguridad Social, en la capital, y siempre que dispongan de un comité de ética bien constituido y registrado en el INS, por lo tanto, no incluyen selectivamente a los pacientes más pobres.

En la carta que firma el distinguido profesor Volnei Garrafa, a quien apreciamos mucho, se dice que: En el Perú no se exige póliza de seguro a favor de los pacientes que

\footnotetext{
1 Instituto de Ética en Salud, Facultad de Medicina, Universidad Nacional Mayor de San Marcos. Lima, Perú.

2 Comité de Ética de Investigación, Asociación PRISMA. Lima, Perú.

3 Comité de Ética en Investigación, Hospital Edgardo Rebagliati Martins. Lima, Perú

a Médico psiquiatra; ${ }^{b}$ médico neurólogo
}

Recibido: 07-03-12 Aprobado: 07-03-12 